\title{
Bohmian mechanics for a degenerate time foliation
}

\author{
Ward Struyve • Roderich Tumulka
}

Received: 12 May 2015 / Accepted: 20 June 2015 / Published online: 16 July 2015

(C) Chapman University 2015

\begin{abstract}
The version of Bohmian mechanics in relativistic space-time that works best, the hypersurface BohmDirac model, assumes a preferred foliation of space-time into spacelike hypersurfaces (called the time foliation) as given. We consider here a degenerate case in which, contrary to the usual definition of a foliation, several leaves of the time foliation have a region in common. That is, if we think of the time foliation as a 1-parameter family of hypersurfaces, with the hypersurfaces moving towards the future as we increase the parameter, a degenerate time foliation is one for which a part of the hypersurface does not move as we increase the parameter. We show that the hypersurface Bohm-Dirac model still works in this situation; that is, we show that a Bohm-type law of motion can still be defined and that the appropriate $|\psi|^{2}$ distribution is still equivariant with respect to this law.
\end{abstract}

Keywords Bohmian mechanics $\cdot$ Foliation $\cdot$ Relativity $\cdot$ Probability flux

\section{Introduction}

The natural extension of Bohmian mechanics to relativistic space-time (flat or curved), known as the hypersurface Bohm-Dirac model [3], makes use of a spacelike foliation of space-time into spacelike hypersurfaces, the "time foliation" $\mathscr{F}$; it can be specified by a 1-parameter family of spacelike hypersurfaces $\Sigma_{t}$, called "time leaves," with $t$ an arbitrary parameter such that for $t_{1} \leq t_{2}, \Sigma_{t_{2}}$ lies in the future of $\Sigma_{t_{1}}$. In terms of arbitrary space-time coordinates $x^{0}, x^{1}, x^{2}, x^{3}, \Sigma_{t}$ can be specified by means of a function $f\left(t, x^{1}, x^{2}, x^{3}\right)$ as the set

$\Sigma_{t}=\left\{\left(x^{0}, x^{1}, x^{2}, x^{3}\right) \mid x^{0}=f\left(t, x^{1}, x^{2}, x^{3}\right)\right\} ;$

the function $f$ is then increasing in the variable $t$, and the property that $\Sigma_{t}$ is spacelike corresponds to certain bounds on the derivatives of $f$ with respect to $x^{1}, x^{2}, x^{3}$.

\footnotetext{
W. Struyve

Department of Physics, Université de Liège, Bâtiment B15, Sart Tilman, 4000 Liège, Belgium

e-mail: ward.struyve@ulg.ac.be

R. Tumulka $(\bowtie)$

Department of Mathematics, Rutgers University, Hill Center, 110 Frelinghuysen Road, Piscataway, NJ 08854-8019, USA

e-mail: tumulka@math.rutgers.edu
} 
Fig. 1 An example of a what we mean by a degenerate foliation (here, of 1+1-dimensional space-time): some of the leaves overlap in a region (here, on the left). The equations defining this particular example are given in "Appendix"

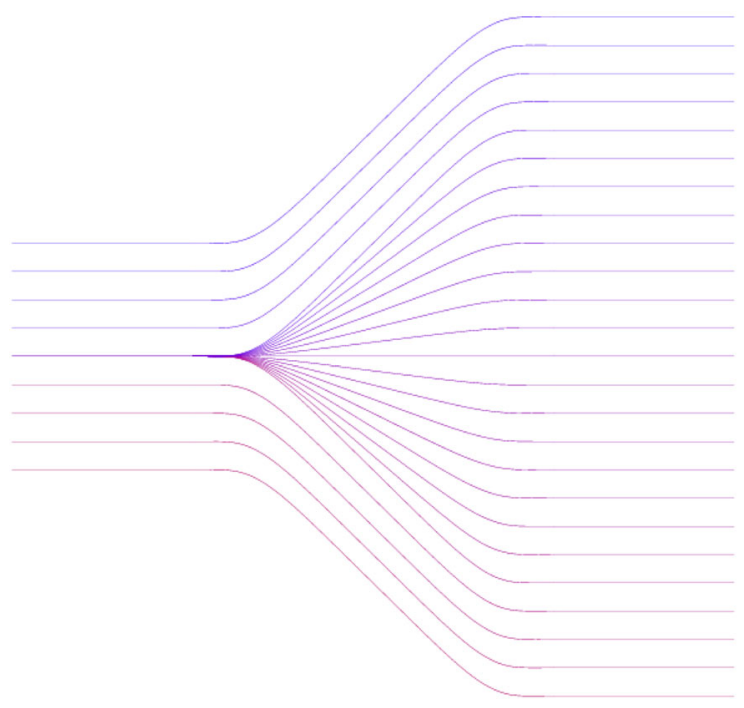

The degenerate case we consider in this note, shown in Fig. 1, corresponds to $f$ having a plateau as a function of $t$, i.e., that $f$ is constant as a function of $t$ on some interval $\left[t_{1}, t_{2}\right]$,

$f\left(t_{1}, x^{1}, x^{2}, x^{3}\right)=f\left(t, x^{1}, x^{2}, x^{3}\right)=f\left(t_{2}, x^{1}, x^{2}, x^{3}\right)$

for all $t \in\left[t_{1}, t_{2}\right]$ and all $\left(x^{1}, x^{2}, x^{3}\right)$ in some region $A \subset \mathbb{R}^{3}$. In particular,

$\frac{\partial f}{\partial t}=0$

for $t \in\left[t_{1}, t_{2}\right]$ and $\left(x^{1}, x^{2}, x^{3}\right)$ in the relevant region $A$. Put differently, when we think of how $\Sigma_{t}$ moves through space-time as we increase the parameter $t$, we allow that a part of $\Sigma_{t}$ does not actually move towards the future but remains constant. That is, there is a piece of hypersurface common to all $\Sigma_{t}$ with $t \in\left[t_{1}, t_{2}\right]$. Note that such a degenerate foliation cannot arise as the level sets of the 0th coordinate function of a space-time coordinate system because some space-time points $x$ lie on several $\Sigma_{t}$ (while any coordinate function would yield a unique value at $x$ ). An explicit example of a degenerate foliation, defined by means of formulas, is provided in "Appendix".

We show here that Bohmian mechanics still works for a time foliation that is degenerate in this sense. More precisely, we show that the definition of the hypersurface Bohm-Dirac model can be extended to this case in such a way that the $|\psi|^{2}$ distribution is still equivariant. We also note that the Bohmian world lines typically have kinks (jump-like changes of direction) when crossing a plateau of $f$, see Fig. 2.

While we are not suggesting that a degenerate time foliation actually occurs in nature, our result is useful for considerations about the flow of probability, which can often be expressed in a particularly intuitive way in terms of Bohmian trajectories. That is, if we think of moving a spacelike hypersurface around in space-time, corresponding to a 1-parameter family $\Sigma_{t}$, then probability will get transported around on $\Sigma_{t}^{N}$ in agreement with the Bohmian motion, and this is still true if the family $\Sigma_{t}$ is degenerate. Thus, our result provides greater freedom in how the hypersurfaces can be moved around, and it is sometimes desirable to push part of the hypersurface to the future while keeping another part unchanged; see [9] for an application of this strategy outside of Bohmian mechanics.

Besides, our result also contributes to illustrating that the hypersurface Bohm-Dirac model is very robust in the sense that it works in many variations of the original setting; previous results in this direction have shown that the hypersurface Bohm-Dirac model also works in curved space-time [14], in space-times with singularities [15], and for hypersurfaces $\Sigma_{t}$ that are not smooth but have kinks [11].

Our result also applies to Bohmian theories with a field ontology instead of a particle ontology and still applies if we drop the assumption $\partial f / \partial t \geq 0$, as we elucidate in Sect. 4. Moreover, also in the case that $\mathscr{F}$ is not everywhere smooth but has kinks, equivariance continues to hold. This phenomenon is discussed in detail in [11] for nondegenerate foliations consisting of surfaces $\Sigma_{t}$ that are manifolds with kinks; the reasons described in [11] apply, 
Fig. 2 The same degenerate foliation as in Fig. 1, along with examples of Bohmian world lines. The latter typically have kinks when crossing a leaf in a region where the time foliation is degenerate (i.e., where leaves overlap)

Fig. 3 Equivariance also holds for this kind of non-smooth, degenerate foliation
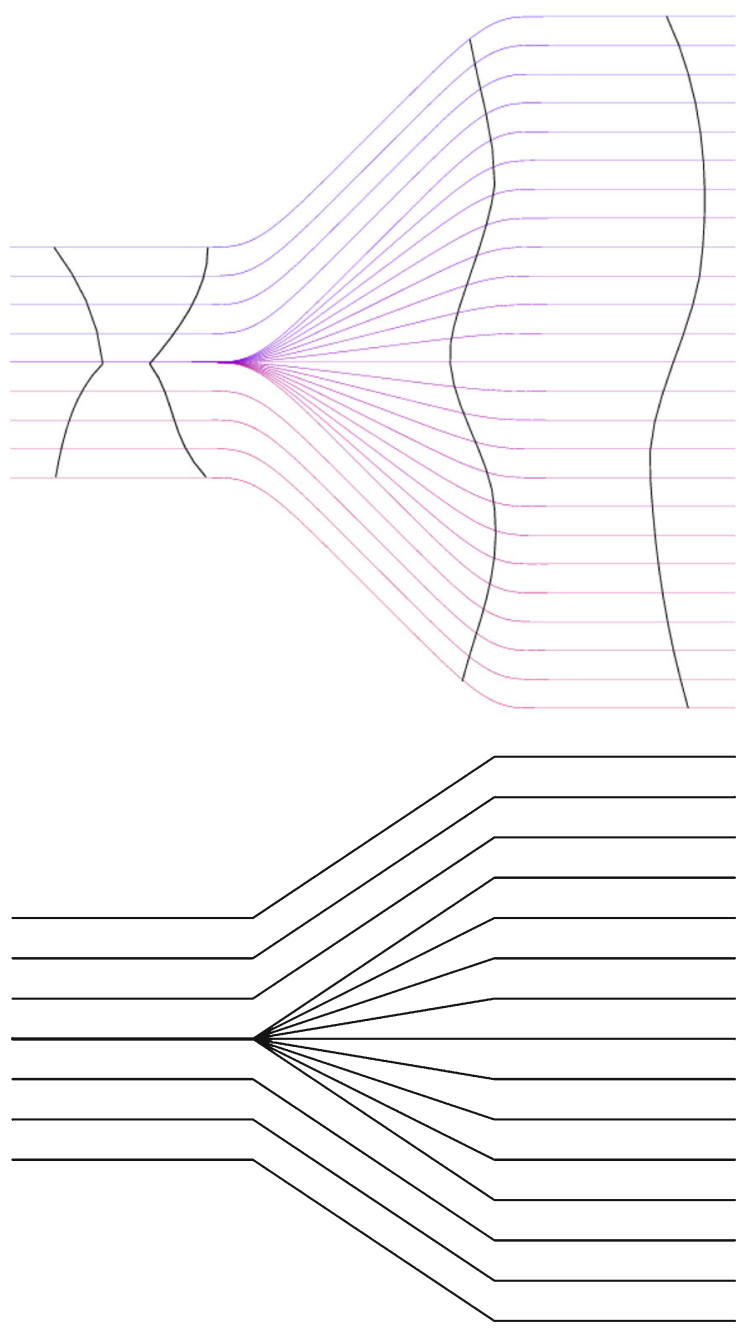

in fact, equally when $\mathscr{F}$ is degenerate and/or when $\mathscr{F}$ involves a (continuous but) non-smooth succession of hypersurfaces. As a consequence, equivariance still holds for $\mathscr{F}$ of the kind depicted in Fig. 3.

This note is organized as follows: in Sect. 2, we recall the definition of the hypersurface Bohm-Dirac model; in Sect. 3, we extend the definition to the degenerate case and show that equivariance is retained; in Sect. 4, we collect some remarks; in "Appendix", we provide an example of a degenerate foliation.

\section{The hypersurface Bohm-Dirac model}

This model is defined for a non-degenerate (spacelike) time foliation $\mathscr{F}=\left\{\Sigma_{t}: t \in \mathbb{R}\right\}$ and $N$ particles as follows [3]. Let $\mathscr{M}$ denote space-time; readers may take this to be Minkowski space-time, but the model works also for a curved space-time. The wave function $\psi: \mathscr{M}^{N} \rightarrow\left(\mathbb{C}^{4}\right)^{\otimes N}$ evolves according to a system of non-interacting multi-time Dirac equations $(c=1=\hbar)$

$i \gamma_{k}^{\mu}\left(\partial_{k \mu}-i e A_{\mu}\left(x_{k}\right)\right) \psi=m_{k} \psi$

for all $k=1, \ldots, N$, with $\partial_{k \mu}=\partial / \partial x_{k}^{\mu}, \gamma_{k}^{\mu}=1 \otimes \cdots \otimes 1 \otimes \gamma^{\mu} \otimes 1 \otimes \cdots \otimes 1$ with $\gamma^{\mu}$ in the $k$ th place, and where $A_{\mu}$ is an external vector potential. Each of the $N$ particles has a world line that is everywhere time- or lightlike (in 
fact [12], almost everywhere timelike, except in very special situations), whose unique intersection point with $\Sigma_{t}$ we denote by $X_{k}(t)$. The law of motion reads

$\frac{\mathrm{d} X_{k}^{\mu}}{\mathrm{d} t} \propto j_{k}^{\mu}=\left(\bar{\psi}\left[\gamma^{\mu_{1}} \otimes \cdots \otimes \gamma^{\mu_{N}}\right] \psi\right)\left(X_{1}(t), \ldots, X_{N}(t)\right) \delta_{\mu_{k}}^{\mu} \prod_{j \neq k} n_{\mu_{j}}\left(X_{j}(t)\right)$

with $n_{\mu}(x)$ the future-pointing unit normal vector to $\Sigma_{t}$ at $x \in \Sigma_{t}$.

For any spacelike hypersurface $\Sigma$, we say "the $|\psi|^{2}$ distribution" for the probability distribution on $\Sigma^{N}$ with density, relative to the Riemannian volume measure on $\Sigma^{N}$ defined by the 3-metric on $\Sigma$, given by

$\rho\left(x_{1}, \ldots, x_{N}\right)=\left(\bar{\psi}\left[\gamma^{\mu_{1}} \otimes \cdots \otimes \gamma^{\mu_{N}}\right] \psi\right)\left(x_{1}, \ldots, x_{N}\right) \prod_{j=1}^{N} n_{\mu_{j}}^{\Sigma}\left(x_{j}\right)$

for all $x_{1}, \ldots, x_{N} \in \Sigma$, with $n_{\mu}^{\Sigma}(x)$ the future-pointing unit normal vector to $\Sigma$ at $x \in \Sigma$. The equivariance property of the hypersurface Bohm-Dirac model asserts that if $\left(X_{1}\left(t_{0}\right), \ldots, X_{N}\left(t_{0}\right)\right)$ is $\left|\psi^{2}\right|$-distributed on $\Sigma_{t_{0}} \in \mathscr{F}$, then $\left(X_{1}(t), \ldots, X_{N}(t)\right)$ is $|\psi|^{2}$-distributed on $\Sigma_{t} \in \mathscr{F}$ for any $t \in \mathbb{R}$.

\section{The hypersurface Bohm-Dirac model for a degenerate time foliation}

We postulate the following law of motion for the version of the model for a degenerate time foliation $\mathscr{F}=\left\{\Sigma_{t}: t \in\right.$ $\mathbb{R}\}$ specified by a function $f$ as in (1), that is, we demand that $f\left(t, \mathbb{R}^{3}\right)$ is a spacelike hypersurface $\Sigma_{t}$ and that $\partial f / \partial t \geq 0$. For any $k$ such that $\mathscr{F}$ is locally non-degenerate at $X_{k}(t)$, i.e., such that

$\frac{\partial f}{\partial t}\left(t, X_{k}^{1}(t), \ldots, X_{k}^{3}(t)\right) \neq 0$,

we keep (5) as the law of motion. For any other $k$, i.e., for any $k$ such that $\mathscr{F}$ is degenerate at $X_{k}(t)$, we set

$\frac{\mathrm{d} X_{k}^{\mu}}{\mathrm{d} t}=0$

which means that we do not move the point $X_{k}$ in space-time when $\Sigma_{t}$ does not move at that point as we increase $t$. This law is the obvious choice, as Eq. (5), with the proportionality factor made explicit, is of the form

$\frac{\mathrm{d} X_{k}^{\mu}}{\mathrm{d} t}=\left(n_{v} j_{k}^{\nu}\right)^{-1} n_{0} \partial_{t} f j_{k}^{\mu}$,

[with $n_{\mu}=(1,-\nabla f) /\left(\sqrt{1-|\nabla f|^{2}}\right)$ ], which vanishes when $\partial_{t} f=0$; thus, Eq. (8) corresponds to keeping (9) also at degeneracies. In other words, our law of motion for a degenerate time foliation is a limiting case of the usual law (5) for a non-degenerate time foliation. Moreover, Eq. (8) is the only possible choice (compatible with our parameterization of the world line defined by the relation $\left.X_{k}(t) \in \Sigma_{t}\right)$ that leads to world lines that are everywhere time- or lightlike. [That is because, as $t$ increases, we cannot have $X_{k}(t+d t)$ in the future of $X_{k}(t)$ if we want it to be on $\Sigma_{t+d t}$, and we cannot have it anywhere on $\Sigma_{t+d t}$ other than at $X_{k}(t)$ if the curve $X_{k}(\cdot)$ can never be spacelike.]

So it is obvious how to choose the law of motion, and the only question is whether this choice leads to equivariance of the $|\psi|^{2}$ distribution. We will show presently that it does. This result is perhaps not surprising, as the law of motion is a limiting case of the law of motion for the non-degenerate case, which is known to lead to equivariance $[3,14]$.

To verify equivariance, we can revisit the equivariance proofs for the hypersurface Bohm-Dirac model given in $[3,14]$ and check that non-degeneracy is not necessary for the proof; we follow here [14]. Suppose that

$\mathscr{C}=\bigcup_{t \in \mathbb{R}} \Sigma_{t}^{N}$ 

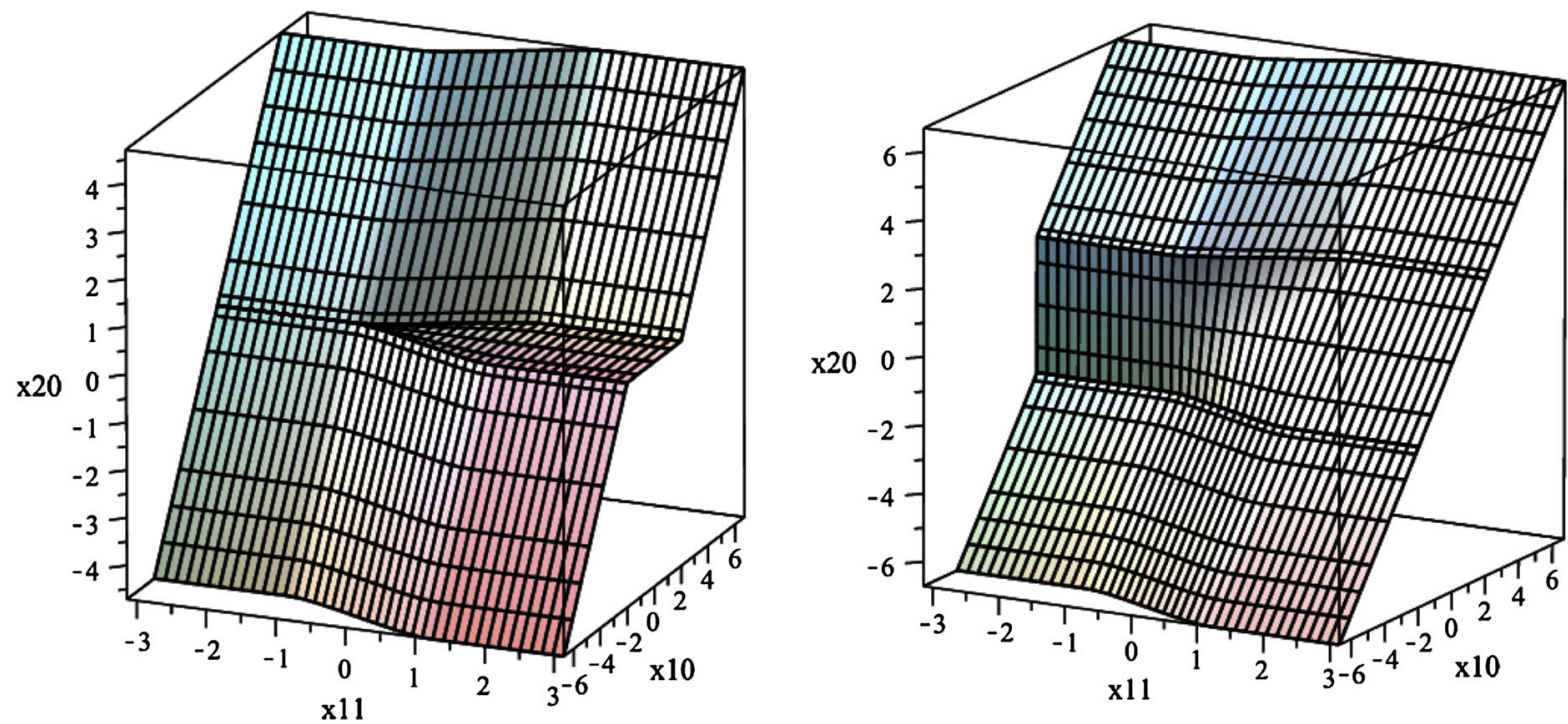

Fig. 4 Two cross-sections of the surface $\mathscr{C}$ as in (10) for the particular foliation shown in Fig. 1 in $1+1$-dimensional space-time for $N=2$ particles; $\mathscr{C}$ is a surface of dimension $(1 N+1)=3$ in $\mathscr{M}^{N}$, which has dimension $(1+1) N=4$. To visualize $\mathscr{C}$, we set the coordinate $x_{2}^{1}=$ const. and display the 2-dimensional surface in 3-dimensional space thus obtained. Left for $x_{2}^{1}$ negative and $\left|x_{2}^{1}\right|$ sufficiently large (in fact, the figure does not depend on the value $x_{2}^{1}$ as long as $x_{2}^{1}<-\pi / 2$ ). Right for $x_{2}^{1}$ positive and sufficiently large

is a piecewise smooth $(3 N+1)$-dimensional surface in $\mathscr{M}^{N}$; see Fig. 4 for an example. (We conjecture that there are degenerate foliations for which $\mathscr{C}$ is smooth rather than merely piecewise smooth, i.e., for which $\mathscr{C}$ has no kinks. ${ }^{1}$ However, our considerations do not require smoothness and work just as well with kinks.)

The probability current tensor defined by the wave function $\psi$,

$j^{\mu_{1}, \ldots, \mu_{N}}\left(x_{1}, \ldots, x_{N}\right)=\bar{\psi}\left[\gamma^{\mu_{1}} \otimes \cdots \otimes \gamma^{\mu_{N}}\right] \psi$,

can be transformed into a $3 N$-form $J$ [14],

$J_{\kappa_{1}} \lambda_{1} \mu_{1} \ldots \kappa_{N} \lambda_{N} \mu_{N}=\varepsilon_{\kappa_{1}} \lambda_{1} \mu_{1} \nu_{1} \ldots \varepsilon_{\kappa_{N} \lambda_{N} \mu_{N} \nu_{N}} j^{\nu_{1} \ldots \nu_{N}}$,

which is closed on $\mathscr{M}^{N}$ and thus also on $\mathscr{C}$. Like any $3 N$-form on a $(3 N+1)$-dimensional manifold, $J$ defines a field of 1-dimensional subspaces $S_{x_{1} \ldots x_{N}}$ on $\mathscr{C}$, its kernels, (except at the points where $J=0$, which are the points where $\psi=0$ ), and the integral curves of $S_{x_{1} \ldots x_{N}}$ are exactly the possible trajectories of the configuration on $\mathscr{C}$ as defined in (5) and (8) above for the degenerate or non-degenerate case. These trajectories satisfy the wandering condition [14] (i.e., they are not closed or almost-closed) because they intersect $\Sigma_{t}^{N}$ only once for every $t$. As a consequence [14], $J$ defines a measure $\mu$ on the set of integral curves of the field $S_{x_{1} \ldots x_{N}}$ that agrees with the $3 N$-form $J$ on any $3 N$-surface in $\mathscr{C}$, and thus in particular on $\Sigma_{t}^{N}$. If $\mathscr{C}$ has kinks, then integral curves need to be extended across kinks, that is, for an integral curve ending at a point $\left(x_{1}, \ldots, x_{N}\right)$ on a kink of $\mathscr{C}$, the integral curve on the other side of the kink starting at $\left(x_{1}, \ldots, x_{N}\right)$ should be regarded as its extension. For almost every kink point, the extension is unique, and $\mu$ is consistently defined on the set of extended integral curves [11]. The measure $\mu$ is normalized (i.e., is a probability measure) because $\psi$ is normalized on each of the $\Sigma_{t}^{N}$ (because it is normalized on $\Sigma^{N}$ for any spacelike Cauchy hypersurface $\Sigma$ in $\mathscr{M}$ ). On surfaces $\Sigma_{t}^{N}, \mu$ is the " $|\psi|^{2}$ distribution" (6), and so this distribution is equivariant.

To sum up, non-degeneracy is not needed for equivariance.

\footnotetext{
${ }^{1}$ We have two reasons for this conjecture. First, it seems that degeneracy, although it violates the standard definition of a foliation, should not disturb the smoothness of $\mathscr{C}$ because degeneracy corresponds to a certain property about the directions tangent to $\mathscr{C}:$ if $\mathscr{F}$ is degenerate around, say, $\Sigma_{t}$ at $x_{k} \in \Sigma_{t}$, then $T_{\left(x_{1} \ldots x_{N}\right)} \mathscr{C} \subseteq T_{x_{k}} \Sigma_{t} \oplus \bigoplus_{j \neq k} T_{x_{j}} \mathscr{M}$, where $T_{x} M$ means the tangent space to the manifold $M$ at the point $x$. The point is that this property has nothing to do with smoothness. Second, we believe that the example in "Appendix" can be so modified as to have smooth $\mathscr{C}$ at the expense of greater complexity of the example.
} 


\section{Remarks}

1. Relation to no-signaling. Our result, that equivariance holds also for degenerate foliations, is related to the wellknown no-signaling theorem as follows. Let $A$ be the region of degeneracy, i.e., the region on the hypersurface $\Sigma_{t}$ that does not move as we increase $t$ (the left region in Fig. 1), and let $A_{t}^{c}$ be its complement in $\Sigma_{t}$ for $t \in\left[t_{1}, t_{2}\right]$. It is a necessary condition for equivariance that the marginal distribution of $\left|\psi_{\Sigma_{t}}\right|^{2}$ for the particles in $A$ does not change as we increase $t$. Indeed, if that condition did not hold, the $|\psi|^{2}$ distribution could not be preserved without moving the particles in $A$. Now that condition is more or less equivalent to the no-signaling theorem: whatever external fields one experimenter chooses in $A_{t}^{c}$ between $\Sigma_{t_{1}}$ and $\Sigma_{t_{2}}$, they have no effect on the distribution of, e.g., pointer particles in $A$. Alternatively, the no-signaling property is often expressed by saying that the reduced density matrix $\rho_{t}^{A}$ for $A$-obtained from the full quantum state on $\Sigma_{t}$ by tracing out $A_{t}^{c}$-does not depend on $t$ between $t_{1}$ and $t_{2}$ for any choice of external fields, $\rho_{t}^{A}=\rho^{A}$. The marginal distribution referred to above is just the distribution with density $\left\langle q\left|\rho^{A}\right| q\right\rangle$, where $q$ is any configuration in $A$, i.e., the density is the diagonal of the position representation of $\rho^{A}$; so the $t$-independence of the marginal distribution follows from the $t$-independence of $\rho^{A}$.

2. Kinks in the world lines (see Fig. 2). While the positions of the particles in the region $A$ of degeneracy do not change as we increase $t$ from $t_{1}$ to $t_{2}$, the velocities may well (and will typically) change, in the sense that $j_{k}^{\mu}$ as in (5) changes with $t$ for particle $k$ in $A$ (unless no particle outside $A$ is entangled with particle $k$ ), and, as a consequence,

$$
\lim _{t \searrow t_{2}}\left[\frac{\mathrm{d} X_{k}^{\mu}}{\mathrm{d} t}\right] \neq \lim _{t \nearrow t_{1}}\left[\frac{\mathrm{d} X_{k}^{\mu}}{\mathrm{d} t}\right],
$$

where $\left[v^{\mu}\right]$ denotes the 1 -dimensional subspace through $v^{\mu}$. [Equivalently, this relation is also true if $\left[v^{\mu}\right.$ ] denotes the unit vector in the direction of $v^{\mu},\left[v^{\mu}\right]=\left(v_{v} v^{\nu}\right)^{-1 / 2} v^{\mu}$, provided that the limiting direction is not lightlike.] This means that the world line of particle $k$ has a kink at the point $X$ where it crosses $A$, with both directions (into $X$ and out of $X$ ) being timelike or lightlike. Particles outside $A$ (i.e., that do not pass through the degeneracy) do not feature kinks.

3. No entering or leaving $A$. Since particles in $A$ do not move, they obviously cannot leave $A$ before $t_{2}$. Conversely, no particle that is outside $A$ on $\Sigma_{t_{1}}$ can enter $A$ between $t_{1}$ and $t_{2}$, as follows from the fact that the world lines are everywhere time- or lightlike.

4. Particle creation and annihilation. We expect that the result of this paper, equivariance of $|\psi|^{2}$ also for degenerate foliations, extends to "Bell-type quantum field theories" [1,5,6], i.e., versions of Bohmian mechanics involving particle creation and annihilation by means of stochastic jumps of the actual configuration in the configuration space of a variable number of particles. These versions are formulated in $[1,5,6]$ for a fixed Lorentz frame in flat space-time. It should be straightforward to adapt them to a non-flat time foliation (also in curved space-time), provided the Hamiltonian can be defined for such a foliation; this will be possible in a natural way for an arbitrary foliation if a multi-time evolution law for the wave function on the set of spacelike configurations is provided. Such laws do not get along with an ultraviolet cut-off; on the formal level, such a law is described and discussed in [8], while on the rigorous level, it may be possible to formulate such a law using interior-boundary conditions [13]. The reason why these theories should work also with degenerate foliations is the following: they have a law specifying the jump rate in terms of the wave function and the interaction Hamiltonian, where "rate" means probability per time and should, therefore, be proportional to the thickness of the layer between $\Sigma_{t}$ and $\Sigma_{t+d t}$ at the point where the particle creation or annihilation occurs. For a degenerate foliation, this thickness will sometimes vanish, and, therefore, no creation or annihilation events should occur at such space-time locations.

5. Field ontologies. There also exist Bohmian approaches with fields as the actual configurations, rather than particle positions (see, e.g., [10]). These approaches could similarly be formulated with respect to a degenerate foliation. For example, for a scalar field, the guidance equation for a non-degenerate time foliation is of the following form [4]: 
Fig. 5 The kind of world lines arising if we allow $\Sigma_{t}$ also to move backwards in time, as explained in

Remark 6

$\frac{\mathrm{d} \varphi(x)}{\mathrm{d} \tau}=\left.\frac{1}{\sqrt{h}} \operatorname{Im}\left(\frac{1}{\Psi_{\Sigma_{x}}} \frac{\delta \Psi_{\Sigma_{x}}}{\delta \varphi_{\Sigma_{x}}(x)}\right)\right|_{\left.\varphi\right|_{\Sigma_{x}}}$,

where $\mathrm{d} \varphi(x) / \mathrm{d} \tau=n^{\mu}(x) \partial_{\mu} \varphi(x)$ is the directional derivative at $x$ along the normal to the time leaf $\Sigma_{x}$ that contains $x, h$ is the determinant of the induced Riemannian metric on $\Sigma_{x},\left.\varphi\right|_{\Sigma_{x}}$ is the restriction of the field configuration to the hypersurface $\Sigma_{x}$, and $\Psi_{\Sigma}\left(\varphi_{\Sigma}\right)=\left\langle\varphi_{\Sigma} \mid \Psi\right\rangle$, where $|\Psi\rangle$ is the state vector in the Heisenberg picture and where $\left|\varphi_{\Sigma}\right\rangle$ is defined by $\varphi(x)\left|\varphi_{\Sigma}\right\rangle=\varphi_{\Sigma}(x)\left|\varphi_{\Sigma}\right\rangle$, for points $x$ on $\Sigma$, with $\varphi(x)$ the Heisenberg field operator. In terms of the parameter $t$ of the time leaves $\Sigma_{t}$, Eq. (14) can be rewritten as

$\frac{\partial \varphi(f(t, \boldsymbol{x}), \boldsymbol{x})}{\partial t}=\frac{\partial f}{\partial t}\left[\left.n_{0} \frac{1}{\sqrt{h}} \operatorname{Im}\left(\frac{1}{\Psi_{\Sigma_{t}}} \frac{\delta \Psi_{\Sigma_{t}}}{\delta \varphi_{\Sigma_{t}}(f(t, \boldsymbol{x}), \boldsymbol{x})}\right)\right|_{\left.\varphi\right|_{\Sigma_{t}}}+u^{\mu} \partial_{\mu} \varphi\right]$,

with $\boldsymbol{x}=\left(x^{1}, x^{2}, x^{3}\right)$ and $u^{\mu}$ the orthogonal projection of the timelike coordinate vector $(1,0,0,0)$ to the tangent plane to $\Sigma_{t}$ at $x=(f(t, \boldsymbol{x}), \boldsymbol{x})$, which can be written as $u^{\mu}=\delta_{0}^{\mu}-n_{0} n^{\mu}$; note that $u^{\mu} \partial_{\mu} \varphi$ is a directional derivative along $\Sigma_{t}$ and thus known if $\varphi_{\Sigma_{t}}$ is given. ${ }^{2}$ If we take (15) as the guidance equation for $\varphi$ also in the case of a degenerate foliation, it implies that, at any point $x=(f(t, \boldsymbol{x}), \boldsymbol{x})$ where the foliation is degenerate (i.e., $\partial f / \partial t=0$ ),

$\frac{\partial \varphi(f(t, \boldsymbol{x}), \boldsymbol{x})}{\partial t}=0$,

in analogy to (8). We expect that equivariance still holds, for the same reasons as for the particle ontology.

6. Generalization to a time foliation going backwards in time. We may even drop the assumption $\partial f / \partial t \geq 0$ and allow any smooth $f$ function such that $f\left(t, \mathbb{R}^{3}\right)$ is a spacelike hypersurface for every $t$. That means that, as we increase $t, \Sigma_{t}$ may move towards the future in some regions, towards the past in others, and remain unchanged elsewhere, with the regions changing with $t$. Again, we are not suggesting that a time foliation like that actually occurs in nature, but it may be worthwhile noticing this mathematical possibility. The law of motion (5) can naturally be adapted to this case by writing it in the form (9), which allows for $\partial_{t} f$ to be positive, negative, or zero. When a world line reaches a point at which $\partial_{t} f$ changes sign, the world line will typically change sign of its direction, as depicted in Fig. 5-an extreme kind of kink.

In this scenario, there is no longer a unique point of intersection between a world line and a time leaf $\Sigma_{t}$; however, the $4 N$ functions $t \mapsto X_{k}^{\mu}(t)$ that together form a (smooth!) solution of the ODE system (9) provide an unambiguous choice of $N$ points on $\Sigma_{t}$ for each $t$. For this configuration, the $|\psi|^{2}$ distribution is again equivariant. Indeed, consider, instead of $\mathscr{C}$, the $(3 N+1)$-dimensional surface

$\overline{2 \text { To verify }(15)}$, note that $\left.\partial_{t} \varphi(f(t, \boldsymbol{x}), \boldsymbol{x})\right)=\partial_{\mu} \varphi \delta_{0}^{\mu} \partial_{t} f$, and $\delta_{0}^{\mu}=(1,0,0,0)=n_{0} n^{\mu}+u^{\mu}$. 


$$
\tilde{\mathscr{C}}=\bigcup_{t \in \mathbb{R}}\{t\} \times \Sigma_{t}^{N}
$$

in the $(4 N+1)$-dimensional space $\mathbb{R} \times \mathscr{M}^{N}$ (which is smooth if $f$ is), and on it the closed $3 N$-form $J$ defined as in (12). Then the kernels of $J$ form again a field of 1-dimensional subspaces on $\tilde{\mathscr{C}}$, the integral curves of which are the solutions of the law of motion (9). Since the $t$ variable is increasing along the integral curves, they obey the wandering condition, and thus [14] the measure defined by $J$ on $\Sigma_{t}^{N}$ (i.e., the $|\psi|^{2}$ distribution) is equivariant.

Acknowledgments Both authors acknowledge support from the John Templeton Foundation, Grant No. 37433. W.S. acknowledges current support from the Actions de Recherches Concertées (ARC) of the Belgium Wallonia-Brussels Federation under Contract No. $12-17 / 02$.

\section{Appendix: Example of a degenerate foliation}

Here is a specific example of an $f$ function such that the foliation it defines according to (1) is degenerate. In fact, this example is depicted in Figs. 1, 2, and 4.

Let $a>0$, and let $g: \mathbb{R} \rightarrow \mathbb{R}$ be a smooth increasing function such that $g(x)=-1$ for $x<-a, g(x)=1$ for $x>a$, and $\mathrm{d} g / \mathrm{d} x \leq 1$ everywhere; for example (depicted in Fig. 6), $a=\pi / 2$ and

$g(x)= \begin{cases}-1 & \text { if } x \leq-\pi / 2 \\ \tanh (\tan (x)) & \text { if }-\pi / 2<x<\pi / 2 \\ 1 & \text { if } \pi / 2 \leq x\end{cases}$

Then we define $f\left(t, x^{1}, x^{2}, x^{3}\right)$ as follows: the function is actually independent of $x^{2}$ and $x^{3}$; for simplicity, we write $x$ instead of $x^{1} ; f(t, x)$ is given by

$f(t, x)= \begin{cases}(t+a) \frac{1}{2}(1-g(t+2 a))-1-g(x) & \text { if } t \leq-a \\ g(t)(1+g(x)) & \text { if }-a \leq t \leq a \\ (t-a) \frac{1}{2}(1+g(t-2 a))+1+g(x) & \text { if } a \leq t .\end{cases}$

The graph of $f$ is shown in Fig. 7. Note the plateau, $f(t, x)=0$ for all $(t, x)$ with $-a \leq t \leq a$ and $x \leq-a$.

Alternatively, if we define

$f_{2}(t, x)= \begin{cases}t+1-g(x) & \text { if } t \leq-2 \\ \frac{1}{2} t(1+g(x)) & \text { if }-2 \leq t \leq 2 \\ t-1+g(x) & \text { if } 2 \leq t\end{cases}$

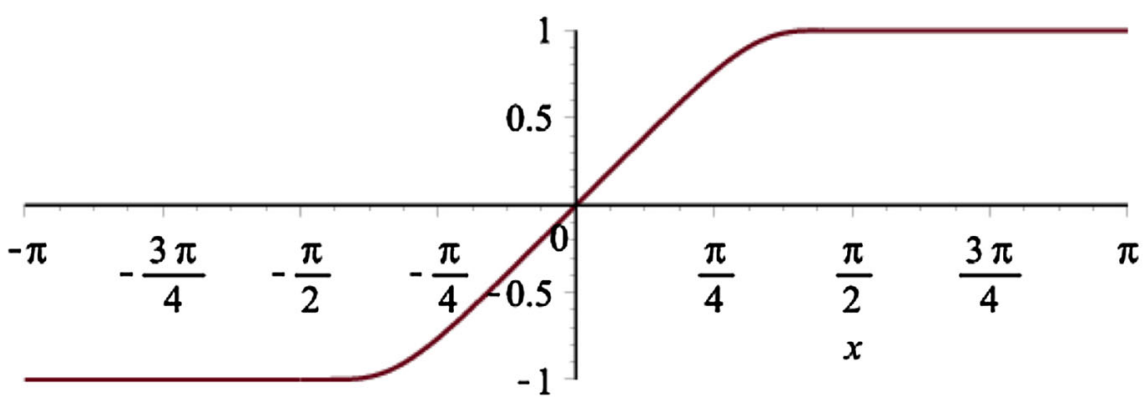

Fig. 6 Graph of the function $g$ defined in (18) 
Fig. 7 Graph of the function $f$ defined in (19). The plateau $f(t, x)=0$ is visible on the left (for $-\pi / 2<t<\pi / 2$ and $x<-\pi / 2$ )

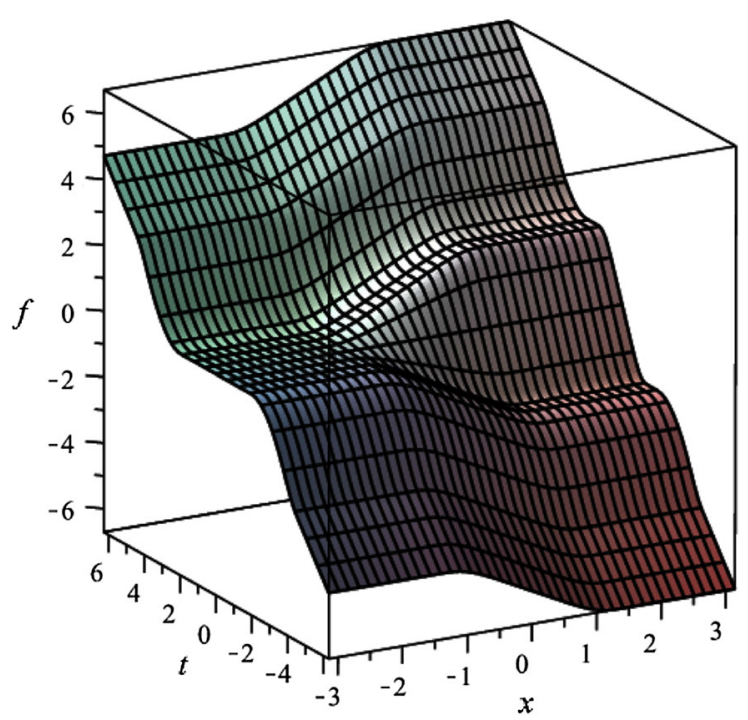

then we actually obtain the same foliation parameterized differently, i.e., the hypersurfaces are labeled differently; the labels are chosen such that $f_{2}(t, x)=t$ for $x>a$. The function $f_{2}$ is not smooth, but the definition is simpler.

The corresponding surface $\mathscr{C}$ is not smooth. For two particles, this can be seen as follows. First note that if $x_{2}^{1}>\pi / 2$, then $f_{2}\left(t, x_{2}^{1}\right)=t$. As such the intersection of $\mathscr{C}$ with a constant $x_{2}^{1}$ hyperplane, which we denote by $\mathscr{C}_{x_{2}^{1}}$, is given by

$\mathscr{C}_{x_{2}^{1}}=\bigcup_{t, x_{1}^{1} \in \mathbb{R}}\left(f_{2}\left(t, x_{1}^{1}\right), x_{1}^{1}, t, x_{2}^{1}\right)=\bigcup_{x_{1}^{1}, x_{2}^{0} \in \mathbb{R}}\left(f_{2}\left(x_{2}^{0}, x_{1}^{1}\right), x_{1}^{1}, x_{2}^{0}, x_{2}^{1}\right)$.

Since $\partial f_{2}(t, x) / \partial t$ is not continuous for $t= \pm 2$ and $x<\pi / 2, \mathscr{C}_{x_{2}^{1}}$ and hence $\mathscr{C}$ are not smooth. This is illustrated in Fig. 4 on the right.

\section{References}

1. Bell, J.S.: Beables for quantum field theory. Phys. Rep. 137, 49-54 (1986). (Reprinted in [2])

2. Bell, J.S.: Speakable and Unspeakable in Quantum Mechanics. Cambridge University Press, Cambridge (1987)

3. Dürr, D., Goldstein, S., Münch-Berndl, K., Zanghì, N.: Hypersurface Bohm-Dirac models. Phys. Rev. A 60, 2729-2736 (1999). arXiv:quant-ph/9801070. (Reprinted in [8])

4. Dürr, D., Goldstein, S., Norsen, T., Struyve, W., Zanghì, N.: Can Bohmian mechanics be made relativistic? Proc. R. Soc. A 470(2162), 20130699 (2014). arXiv:1307.1714

5. Dürr, D., Goldstein, S., Tumulka, R., Zanghì, N.: Bohmian mechanics and quantum field theory. Phys. Rev. Lett. 93, 090402 (2004). arXiv:quant-ph/0303156. (Reprinted in [8])

6. Dürr, D., Goldstein, S., Tumulka, R., Zanghì, N.: Bell-type quantum field theories. J. Phys. A Math. Gen. 38, R1-R43 (2005). arXiv:quant-ph/0407116

7. Dürr, D., Goldstein, S., Zanghì, N.: Quantum Physics Without Quantum Philosophy. Springer, Heidelberg (2013)

8. Petrat, S., Tumulka, R.: Multi-time wave functions for quantum field theory. Ann. Phys. 345, 17-54 (2014). arXiv:1309.0802

9. Petrat, S., Tumulka, R.: Physical significance of multi-time wave functions (2015). (in preparation)

10. Struyve, W.: Pilot-wave theory and quantum fields. Rep. Prog. Phys. 73, 106001 (2010). arXiv:0707.3685

11. Struyve, W., Tumulka, R.: Bohmian trajectories for a time foliation with kinks. J. Geom. Phys. 82, 75-83 (2014). arXiv:1311.3698

12. Tausk, D.V., Tumulka, R.: Can we make a Bohmian electron reach the speed of light, at least for one instant? J. Math. Phys. 51, 122306 (2010). arXiv:0806.4476

13. Teufel, S., Tumulka, R.: New type of Hamiltonians without ultraviolet divergence for quantum field theories (2015). arXiv:1505.04847 
14. Tumulka, R.: Closed 3-forms and random world lines. Ph.D. dissertation, Mathematics Institute, Ludwig-Maximilians University Munich, Germany. http://edoc.ub.uni-muenchen.de/7/ (2001). Accessed 11 May 2015

15. Tumulka, R.: Bohmian mechanics at space-time singularities. II. Spacelike singularities. Gen. Relativ. Gravit. 42, 303-346 (2010). arXiv:0808.3060 